\title{
Ultrathin channels make transistors go faster
}

\section{Reducing the thickness of an amorphous conductive indium tin oxide layer down to a few nanometers has enabled the realization of $40-\mathrm{nm}$-Iong channel transistors with remarkable operating characteristics.}

\section{Thomas D. Anthopoulos}

Many of our modern day electronics are slowly diverging from their traditional forms to new shapes and sizes creating new technology strands. ${ }^{[1-5]}$ One example is the large-area electronics that, unlike traditional integrated circuits (ICs), exploit semiconductors that can be processed over a broad range of large-size substrates, including flexible, stretchable, or transparent ones, for a variety of emerging applications. ${ }^{[2-5]}$ Historically, these technologies have been demarcated by the level of performance by their common building block - the field-effect transistor. State-of-the-art ICs rely mostly on silicon (Si) transistors that combine superb operating characteristics with small footprint both enabled by continuous channel downscaling. ${ }^{[2]}$ In contrast, large-area electronics exploit transistor technologies based on semiconductors with relatively modest performance but that can be manufactured reliably over large substrates. ${ }^{[3-5]}$

Key operating parameters for field-effect transistors include charge carrier mobility, operating voltage, minimum and maximum attainable channel currents, and operating speed. To this end, the charge carrier mobility is ultimately determined by the intrinsic properties of the semiconductor. The speed of operation is governed by the transit time of injected carriers which in turn depends on both the transistors' channel length and the carrier mobility. The shorter the channel and the larger the mobility are, the faster the transistors can be switched on and off. However, combining nanometer-size channel transistors with easy to process semiconductors is challenging making the realization of high frequency operating transistors a difficult task.

Writing in Nature Materials, Shengman Li and co-workers report transistors based on ultrathin amorphous indium tin oxide (ITO) whose channel length ( $L_{c h}$ ) can be aggressively downscaled to $40 \mathrm{~nm}$ (Fig. 1), allowing operation at frequencies in excess of $10 \mathrm{GHz}{ }^{[6]}$ Key to such development is the minimization of detrimental short-channel effects (SCE) that usually prevent such small transistors to be switched off. The researchers showed that the high electron concentration in amorphous conductive ITO films thicker than $10 \mathrm{~nm}$ made turnoff difficult; in contrast, reducing the channel thickness $t_{\text {channel }}$ to $4 \mathrm{~nm}$ and combining it with a $5 \mathrm{~nm}$-thick La- 
doped hafnium-oxide ( $\mathrm{HfLaO}$ ) gate dielectric was found to suppress the electron concentration leading to efficient transistor operation.

These ultrathin ITO transistors can be easily processed via radio frequency (RF) sputtering (making them compatible to large-area fabrication), require a low thermal budget during fabrication and sustain a high electron mobility $\left(>50 \mathrm{~cm}^{2} / \mathrm{Vs}\right)$, consistent with previous studies. ${ }^{[7-}$

8] $\mathrm{Li}$ and colleagues argue that the surprising immunity of electron transport to SCE is due to a combination of factors, including the low permittivity $(\varepsilon)$ of ITO $(\approx 4)$ when compared to traditional semiconductors such as Si (11.7). The low $\varepsilon$ of ITO, when combined with the geometry and physical properties of HfLaO, results to a very small natural length $(\lambda)$ - which is the distance at which the electric field lines from the source and drain electrodes extend into the channel - of $1.8 \mathrm{~nm}$. Transistors with $L_{c h}$ several times longer than $\lambda$ are virtually free of SCE; as a result, ITO has the potential to enable the fabrication of transistors with $L_{c h}$ below $5 \mathrm{~nm}$, making the technology relevant for high performance electronics. ${ }^{[6]}$

Benchmarking the amorphous ITO transistors with other promising materials, a few interesting aspects emerge. The immunity of ultrathin ITO transistors to SCE appears to be superior to both $\mathrm{Si}$ and two-dimensional (2D) semiconductors, leading to exceptionally high channel currents of $520 \mu \mathrm{A} / \mu \mathrm{m}$. The large bandgap of the $4 \mathrm{~nm}$-thin ITO $(\approx 3.7 \mathrm{eV})$ also plays a critical role in suppressing the off current to exceptionally low levels of $100 \mathrm{fA} / \mu \mathrm{m}$, leading to the remarkably high on/off current ratio of $5.5 \times 10^{9}$. The subthreshold slope (SS) remains very low (66 $\mathrm{mV} / \mathrm{dec}$ ) even in transistors with $L_{c h}=40 \mathrm{~nm}$. These excellent operating characteristics yield fast transistors when assessed via RF measurements. A maximum cut-off frequency $f_{\mathrm{T}}$ (defined as the frequency at which the ratio of drain to source and gate to source current is 1) of $10 \mathrm{GHz}$ was measured for $40 \mathrm{~nm}$ channel ITO transistors, outperforming even 2D semiconductor-based transistors. Going a step further, the researchers integrated two ITO transistors to realise lowvoltage unipolar enhancement-depletion mode inverter circuits. High signal gains in the range 178 to 467 were obtained further showcasing the potential of the ultrathin body ITO technology for practical applications.

Understanding the apparent immunity of the amorphous ultrathin body ITO transistors to SCE together with device engineering would be critical for pushing the technology further but also understanding its limitations. It also remains to be seen whether other metal oxides or multilayer oxide systems exhibit properties similar to ITO. The results shown by Li and colleagues are a promising starting point for the further exploration of conductive metal oxides in high-performance, large-area electronics. 
Thomas D. Anthopoulos

King Abdullah University of Science and Technology (KAUST), KAUST Solar Center (KSC), Thuwal 23955, Saudi Arabia.

Email: thomas.anthopoulos@kaust.edu.sa

\section{REFERENCES}

[1] Gupta, S., Navaraj, W.T., Lorenzelli, L \& Dahiya, R., npj Flexible Electronics, 2, 8 (2018).

[2] Franklin, A. D. Science 349, aab2750 (2015).

[3] Wu, W. Nanoscale, 9, 7342-7372 (2017).

[4] Khan, S., Lorenzelli, L. \& Dahiya, R.S. IEEE Sensors Journal, 15, 3164-3185 (2015).

[5] Petti,L. et al., Applied Physics Reviews 3, 021303 (2016).

[6] Li,S. et al., Nature Materials 18, xxx-xxx (2019).

[7] Faber, H. et al., Science Advances 3, e1602640 (2017).

[8] Shih, C. W., Chin, A., Lu, C.F. \& Su, W.F. Scientific Reports, 6, 19023 (2016).
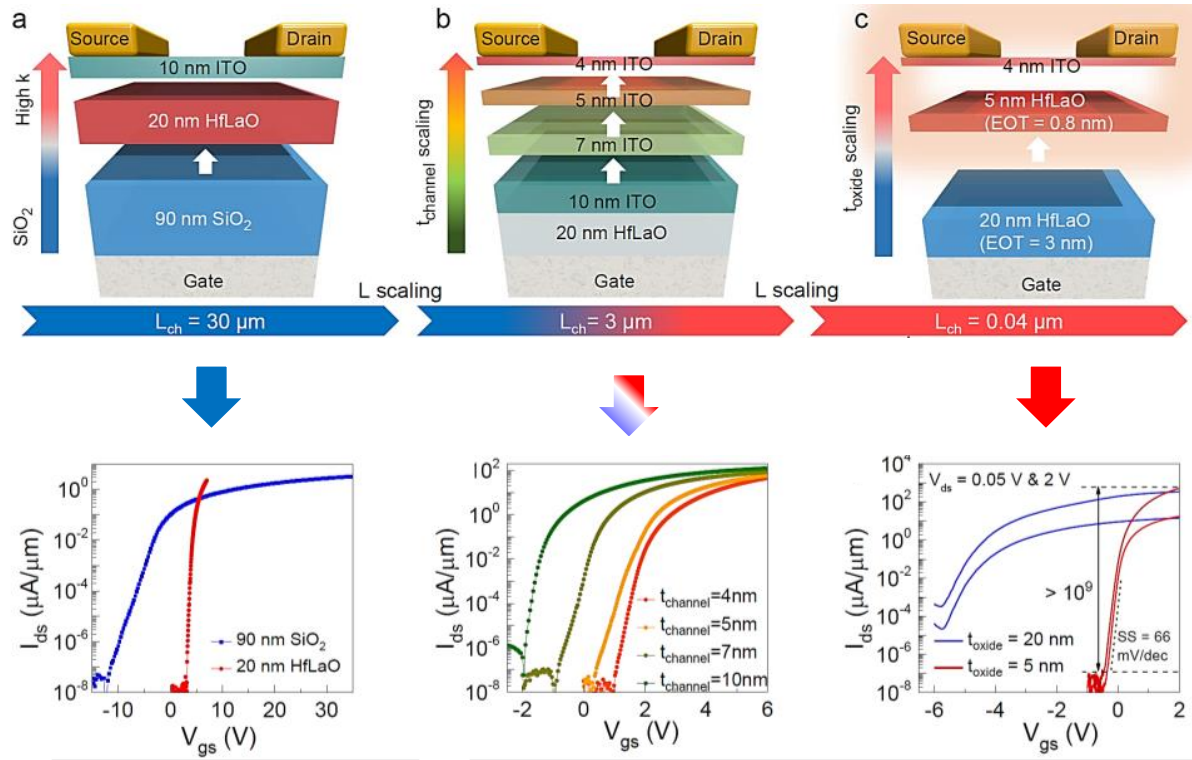

Fig. 1| Transistor structure and the impact of materials and parameters scaling on operating characteristics. Illustration of transistors (top), and the corresponding transfer characteristics (bottom), with; (a) a 30- $\mu \mathrm{m}$-long channel ( $\left.L_{c h}\right)$ employing a 10-nm-thick ITO, and a $20-\mathrm{nm}-\mathrm{HfLaO}$ or $90-\mathrm{nm}-\mathrm{SiO}_{2}$, (b) a transistor with $L_{c h}=3 \mu \mathrm{m}$ and a $20 \mathrm{~nm}$-thick $\mathrm{HfLaO}$ dielectric employing ITO channels with varying thickness between 4 to $10 \mathrm{~nm}$, and (c) a transistor with $L_{c h}=40 \mathrm{~nm}$ and 4-nm-thick ITO channel combined with either a 5-nm-thick HfLaO or 20-nm-HfLaO as the gate dielectric. Adapted from ref. 6, Springer Nature Ltd. 